Annuaire suisse de politique de développement

\title{
Les facteurs déterminant le choix des pays bénéficiaires de l'aide privée suisse
}

\section{Christoph Stamm}

\section{(2) OpenEdition}

12 Journals

Édition électronique

URL : http://journals.openedition.org/aspd/453

DOI : 10.4000/aspd.453

ISSN : 1663-9669

Éditeur

Institut de hautes études internationales et du développement

\section{Édition imprimée}

Date de publication : 1 novembre 2004

Pagination : 83-98

ISSN : 1660-5934

\section{Référence électronique}

Christoph Stamm, «Les facteurs déterminant le choix des pays bénéficiaires de l'aide privée suisse », Annuaire suisse de politique de développement [En ligne], 23-2 | 2004, mis en ligne le 08 mars 2010 consulté le 08 septembre 2020. URL : http://journals.openedition.org/aspd/453 ; DOI : https://doi.org/ 10.4000/aspd.453 


\title{
Les facteurs déterminant le choix des pays bénéficiaires de l'aide privée suisse
}

\author{
Christoph Stamm*
}

\section{Introduction}

Ces dernières années, l' «aide à l'étranger» provenant de fonds privés suisses a atteint entre 280 et 330 millions de francs ${ }^{1}$. Ce total englobe la coopération au développement, l'aide humanitaire et l'aide aux pays en transition. Cet argent est utilisé par des œuvres d'entraide suisses et par d'autres organisations non gouvernementales (ONG) pour réaliser des projets - presque exclusivement bilatéraux - dans les pays en développement et en transition. Contrairement à l'aide publique, l'aide privée n'est pas coordonnée et organisée par un service central. L'aide privée est en effet décentralisée: elle est planifiée et mise en œuvre par quelques grandes œuvres d'entraide et d'innombrables petites $\mathrm{ONG}^{2}$.

Il est intéressant de se demander si la multitude des décisions que prennent isolément ces diverses organisations privées pour choisir leurs projets et les pays ou régions qui en bénéficient débouche sur quelque chose de cohérent. Nous partons ici de l'idée que les montants de l'aide privée ne sont pas répartis uniformément (selon le principe de l'arrosoir) entre les pays en développement et en transition, mais que les moyens à disposition - de toute évidence limités - sont attribués aux pays du Sud et de l'Est sur la base de priorités clairement établies. La présente analyse a donc pour objectif d'identifier les principaux facteurs qui président au choix des pays bénéficiaires de l'aide privée au développement.

Voici notre hypothèse de départ: la répartition géographique de l'aide privée de la Suisse subit l'influence de deux catégories de facteurs. La première catégorie comprend des facteurs propres aux pays en développement et extérieurs à la Suisse, tels que la situation économique et sociale d'un pays, sa situation humanitaire et sa place dans la communauté des pays bénéficiaires. La seconde catégorie de facteurs regroupe les facteurs internes, c'est-à-dire liés à la Suisse. On y trouve la répartition de l'aide publique au développement, la population résidante provenant de pays en développement et en transition ou l'éloignement géographique du pays bénéficiaire. Dans notre analyse, nous accordons une attention particulière au rapport entre aide privée et coopération publique au développement, car nombre d'indices donnent à penser que cette dernière exerce une grande influence sur l'aide privée.

L'initiative individuelle étant à l'origine de nombreux projets, nous admettons toutefois aussi que le facteur «préférences individuelles» joue un rôle non négligeable dans la répartition géographique.

* Politologue.

1 Les œuvres d'entraide et ONG ne fournissant pas toutes des indications sur les montants qu'elles consacrent à la coopération au développement, la somme globale de l'aide privée au développement doit en réalité être plus élevée.

2 Sur la structure des ONG suisses, voir l'article de Gérard Perroulaz dans cet Annuaire (p. 9-24). 


\section{Modèle de base}

Facteurs externes et propres aux pays bénéficiaires (dont les "aspects humanitaires») Facteurs propres à la Suisse

(dont I'«aide publique»)

Préférences individuelles

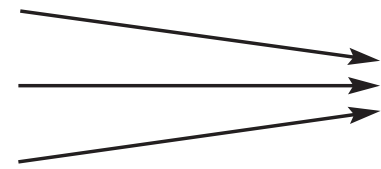

Répartition géographique

de l'aide privée suisse

au développement

Cette analyse quantitative se fonde sur les montants privés versés par la Suisse en 2001 et prend en compte 150 pays en développement et en transition comptant au moins 200'000 habitants. Dans la partie suivante, nous obtiendrons ainsi une esquisse de la répartition géographique de l'aide privée. Nous procéderons ensuite à l'examen des diverses hypothèses partielles, afin d'obtenir, pour terminer, un modèle global éprouvé qui nous fournira des informations sur les différents facteurs déterminant la répartition géographique de l'aide privée.

Répartition et concentration géographiques

\section{Répartition par pays}

En 2001, l'aide privée a alloué 330,6 millions de francs à des pays en développement et en transition. Sur ce montant, 296 millions (90\% environ) ont bénéficié à des pays déterminés et 34,6 millions (10\% environ) ont été versés à des projets internationaux et ne peuvent donc être attribués à un pays précis. Au total, 127 pays ont bénéficié des fonds privés provenant de Suisse et 65 d'entre eux ont reçu plus d'un million de francs. Le tableau 1 met en évidence de grosses différences entre les montants versés et indique les vingt principaux pays bénéficiaires, qui drainent plus de $60 \%$ de l'aide privée suisse ${ }^{3}$. Il permet par ailleurs de constater que six pays reçoivent ensemble plus d'un tiers des fonds distribués.

Pour pouvoir estimer si le niveau des montants s'explique par la taille du pays, il vaut la peine de les mettre en rapport avec le nombre d'habitants. C'est pourquoi le tableau 2 présente les principaux pays bénéficiaires en tenant compte de leur population. La présentation de l'aide par habitant met en évidence l'aide financière dont bénéficient la population et chaque habitant. Dans cette présentation, les pays les plus peuplés ne figurent plus parmi les principaux bénéficiaires. Neuf pays (en gras dans les tableaux) comptent parmi les vingt premiers dans ces deux classements différents. Cependant, même le second mode de classement ne gomme pas les énormes différences entre pays bénéficiaires.

Bien que l'aide soit répartie parmi 127 des 150 pays en développement et en transition, on constate une concentration relativement marquée sur un nombre restreint de pays. On peut en déduire que les œuvres d'entraide privées se fixent des priorités lorsqu'elles choisissent les pays bénéficiaires. Nous aborderons les facteurs qui déterminent cet ordre des priorités dans le chapitre «Facteurs influant sur le choix des pays ».

3 Les places 21 à 30 sont occupées par les pays suivants: Guatemala, Roumanie, Bangladesh, Kenya, Haïti, Cameroun, Burkina Faso, Vietnam, Tchad et Ouganda. 
Tableau 1: Les vingt principaux pays bénéficiaires de l'aide privée suisse, 2001 (en chiffres absolus)

\begin{tabular}{lccc}
\hline Pays & $\begin{array}{c}\text { Aide privée } \\
\text { (milliers de fr.) }\end{array}$ & $\begin{array}{c}\text { Aide privée } \\
\text { (en pourcentage) }\end{array}$ & $\begin{array}{c}\text { Aide privée } \\
\text { (en pourcentage cumulé) }\end{array}$ \\
\hline 1. Yougoslavie & 27200 & 9.2 & 9.2 \\
\hline 2. Inde & 21646 & 7.3 & 16.5 \\
\hline 3. Cambodge & 19959 & 6.7 & 23.2 \\
\hline 4. Mozambique & 11005 & 3.7 & 27.0 \\
\hline 5. Soudan & 10659 & 3.6 & 30.6 \\
\hline 6. Brésil & 8455 & 2.9 & 33.4 \\
\hline 7. Honduras & 7047 & 2.4 & 35.8 \\
\hline 8. Colombie & 6913 & 2.3 & 38.1 \\
\hline 9. Tanzanie & 6712 & 2.3 & 40.4 \\
\hline 10. Nicaragua & 5897 & 2.0 & 42.4 \\
\hline 11. Ethiopie & 5885 & 2.0 & 44.4 \\
\hline 12. Albanie & 5825 & 2.0 & 46.4 \\
\hline 1. El Salvador & 5661 & 1.9 & 50.3 \\
\hline 14. Bolivie & 5562 & 1.9 & 52.0 \\
\hline 15. Pérou & 5472 & 1.8 & 53.7 \\
\hline 16. Philippines & 5169 & 1.7 & 55.5 \\
\hline 17. Afghanistan & 5091 & 1.7 & 57.2 \\
\hline 18. Turquie & 5036 & 1.7 & 58.8 \\
\hline 19. Bosnie-Herzégovine & 4834 & 1.6 & 100.3 \\
\hline 20. Congo, Rép. démocratique & 4458 & 1.5 & $\mathbf{1 0 0 . 0}$ \\
\hline Autres pays & 117492 & $\mathbf{1 0 0 . 0}$ & \\
\hline Total & $\mathbf{2 9 5 9 7 8}$ & & \\
\hline Aide attribuée par pays & 1973 & & \\
\hline Aide bilatérale totale par pays & 2204 & & \\
\hline & & & \\
\hline
\end{tabular}

Tableau 2: Les vingt principaux bénéficiaires de l'aide privée suisse (par habitant)

\begin{tabular}{lrlc}
\hline Pays & Montant de l'aide (ct./hab.) & Pays & Montant de l'aide (ct./hab.) \\
\hline 1. Yougoslavie & 257 & 11. Bolivie & 67 \\
\hline 2. Albanie & 188 & 12. Mozambique & 60 \\
\hline 3. Cambodge & 152 & 13. Palestine (Territoires occupés par Israël) & 54 \\
\hline 4. Bosnie-Herzégovine & 124 & 14. Liban & 51 \\
\hline 5. Nicaragua & 116 & 15. Haïti & 48 \\
\hline 6. Honduras & 110 & 16. Arménie & 47 \\
\hline 7. Gabon & 97 & 17. Swaziland & 45 \\
\hline 8. El Salvador & 90 & 18. Tchad & 42 \\
\hline 9. Bhoutan & 71 & 19. Erythrée & 39 \\
\hline 10. Mauritanie & 71 & 20. Guatemala & 38 \\
\hline Aide attribuée par habitant & & & 5.69 \\
\hline Aide bilatérale totale par habitant & & & 6.36 \\
\hline
\end{tabular}

\section{Répartition par continents}

Le graphique 1 illustre la répartition géographique de l'aide privée par continents. L'Afrique et l'Asie reçoivent près d'un tiers des fonds, les pays en développement du continent américain plus du cinquième et un peu plus du sixième est destiné à l'Europe. Entre le continent qui reçoit le moins d'aide (l'Europe) et celui qui en reçoit la plus grande part (l'Afrique), le rapport est de 1 à 2 . Si nous classons à présent les montants attribués en fonction de la population des pays 
bénéficiaires, nous obtenons un tout autre résultat (graphique 2). Les habitants des pays en développement ou en transition européens, africains et américains se voient attribuer entre 12 et 13 centimes par personne, tandis que ceux des pays d'Asie ne reçoivent que 2,6 centimes par personne. Cela montre que l'Asie occupe une place beaucoup moins grande dans l'aide privée que les autres continents. Dans ce second classement, c'est en effet l'Europe qui profite le plus de l'aide privée: le rapport entre l'Asie et l'Europe est de 1 à 5 .

\section{Graphique 1: Répartition de l'aide privée par continents (en pourcentage)}

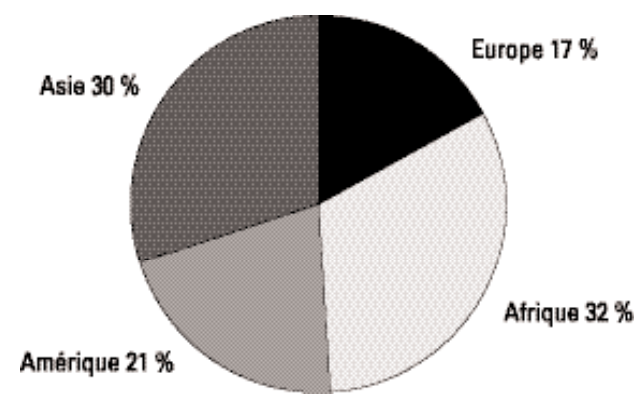

Graphique 2: Aide privée par habitant (en centimes)

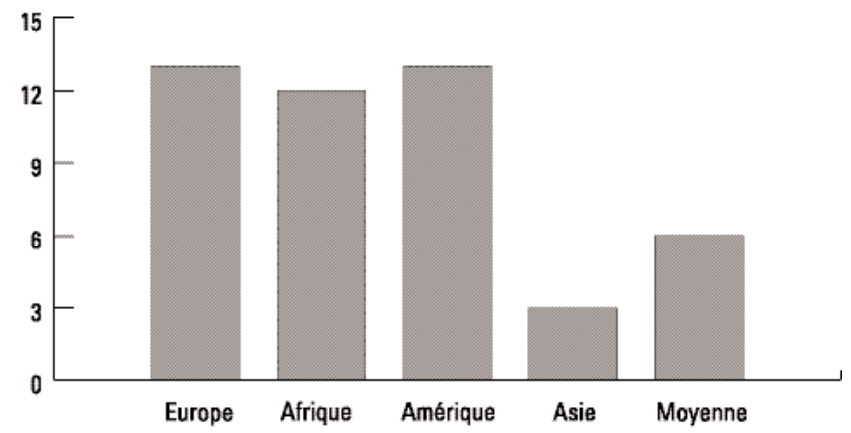

\section{Répartition par catégories de revenu}

Considérer la répartition de l'aide privée en fonction du revenu moyen par habitant dans les pays considérés fournit les premières indications sur les éventuelles priorités appliquées par les œuvres d'entraide privées. Pour ce faire, nous avons utilisé les catégories de revenu définies en 2001 par l'OCDE ${ }^{4}$. La Chine et l'Inde, présentées séparément dans les graphiques ci-après en raison de leur population et pour faciliter la lecture des graphiques, comptent parmi les pays à faible revenus.

4 «Liste des pays bénéficiaires de l'aide établie par le CAD (2001) », Annuaire suisse de politique de développement 2004, vol. 23, n⿳0 1, pp. 335-336.

5 On peut donc aussi comparer l'Inde et la Chine avec les graphiques 1 et 2 (répartition de l'aide privée par continents). 
Le graphique 3 montre que les deux catégories de pays «à faible revenu » et «à revenu intermédiaire - tranche inférieure» se partagent plus de $80 \%$ de l'aide. Le graphique 4 indique, quant à lui, le montant de l'aide par habitant pour chacune des catégories de revenu et souligne que ce sont les pays de la catégorie «à revenu intermédiaire - tranche inférieure » qui sont les principaux bénéficiaires de l'aide privée en provenance de Suisse. Si la Chine et l'Inde n'étaient pas présentées séparément, mais dans leur catégorie, c'est-à-dire celle des pays «à faible revenu», le montant de l'aide par habitant pour cette catégorie de pays aurait diminué pour se situer à 4,7 centimes, soit pratiquement au niveau de l'aide allouée aux pays «à revenu intermédiaire - tranche supérieure». Cet aperçu permet de conclure que l'aide privée ne se concentre ni sur les pays en développement les plus pauvres ni sur les plus riches, mais plutôt sur les pays à revenu moyen.

\section{Graphique 3: Répartition de l'aide privée par catégorie de revenu (en pourcentage)}

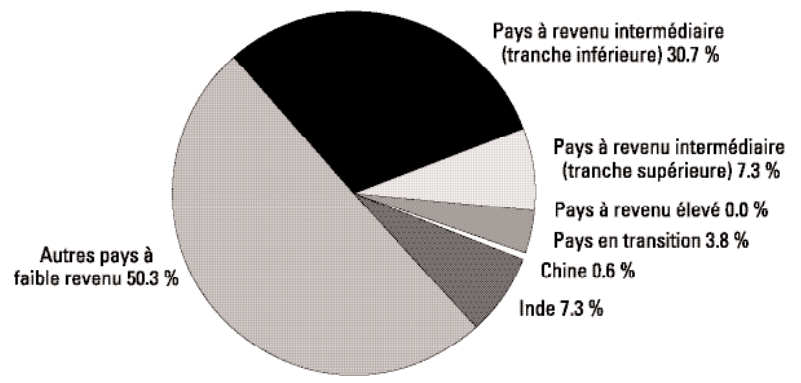

Graphique 4: Aide privée par habitant selon la catégorie de revenu (en centimes)

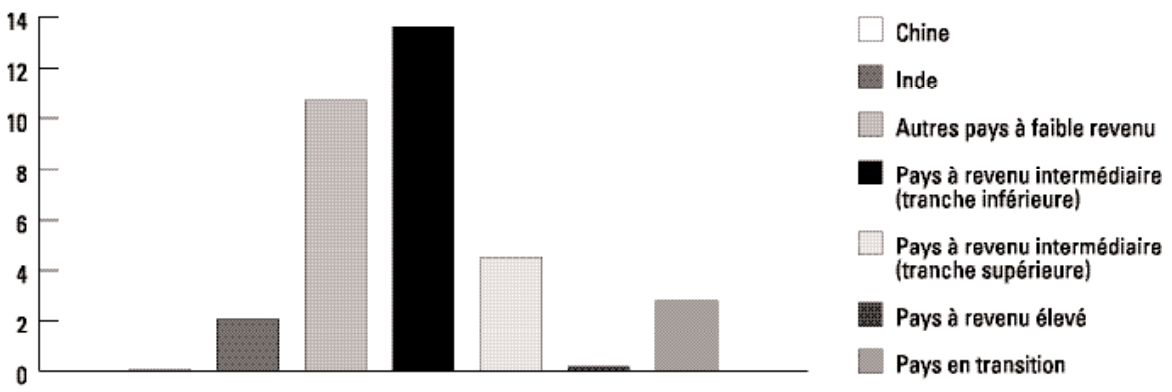

\section{Synthèse}

Nous avons constaté que les fonds sont destinés à un grand nombre de pays, que les montants alloués par pays varient grandement et que $50 \%$ de l'aide se concentrent sur quatorze pays. Nous avons vu par ailleurs que l'Asie, qui est le continent le plus peuplé, bénéficie d'une aide moindre par rapport à sa population que les autres continents. Enfin, nous avons perçu une concentration de l'aide sur les pays en développement à revenu intermédiaire. Dans la partie ciaprès, nous allons expliquer nos hypothèses de base sur la répartition de l'aide privée et tester leur validité. 


\section{Facteurs externes et propres aux pays bénéficiaires}

\section{$\square$ Niveau de développement d'un pays}

Cette hypothèse se fonde sur l'idée que les caractéristiques des pays en développement déterminent la répartition géographique de l'aide. La première de ces caractéristiques est le niveau de développement. Une coopération au développement qui se veut cohérente et qui vise en priorité à lutter contre la pauvreté apporterait en toute logique un soutien accru aux pays les plus pauvres. Voici l'hypothèse partielle qui en ressort: «Moins un pays est développé, plus grands seront les montants que lui allouera l'aide privée suisse.» Pour connaître le niveau de développement, nous utilisons l' «indice de développement humain» des Nations unies, qui mesure le «bien-être» des habitants. Comme le laissait supposer la répartition par catégorie de revenu, présentée ci-dessus, l'analyse de régression linéaire ne révèle pas de relation significative entre le niveau de développement humain d'un pays et l'aide privée de la Suisse ${ }^{6}$. L'hypothèse n'est donc pas valable.

\section{$\square$ Situations de détresse et aspects humanitaires}

Les œuvres d'entraide dépendent des dons qu'elles reçoivent, de sorte que l'orientation de leur coopération au développement tient compte, en partie du moins, des préférences de leurs donateurs. Cette hypothèse partielle part de l'idée que les situations de détresse provoquées par des guerres, des catastrophes naturelles ou des mauvaises récoltes sont plus à même d'inciter les gens à verser un don $^{7}$. Plus la catastrophe humanitaire est grave, plus les montants alloués à la région touchée sont élevés. Il est vrai aussi que l'aide humanitaire représente entre un quart et un tiers de l'aide privée, soit une part plus grande que dans l'aide publique.

Pour mesurer la gravité des conflits armés dans notre analyse, nous utilisons comme indicateur le nombre des victimes estimé pour les années 1997 à 2001 par l'Institut international de recherche sur la paix (SIPRI), en Suède. Pour évaluer la gravité des catastrophes naturelles, nous avons opté pour les indications concernant les victimes fournies pour la période 1998 à 2001 par la Société suisse de réassurance (SwissRe). Enfin, comme indicateur global de la situation humanitaire, nous avons considéré le nombre des personnes sous-alimentées de 1999 à 2001 selon les données de la FAO (Organisation des Nations unies pour l'alimentation et l'agriculture $)^{8}$.

L'analyse par corrélation montre qu'il existe un rapport statistiquement significatif entre les «indicateurs de catastrophes» et la répartition géographique de

6 Voir aussi l'annexe. Les variables «démocratie et droits de l'homme» (indicateur de la Freedom House) et «bonne gouvernance» (indicateur de la Banque mondiale) n'exercent aucune influence sur le choix des pays.

7 Les journées nationales de la Chaîne du Bonheur organisées au lendemain de grandes catastrophes (en 1998 pour les victimes de l'ouragan Mitch qui avait déferlé sur l'Amérique centrale, en 1999 pour les victimes du tremblement de terre en Turquie et pour les victimes de la guerre en Yougoslavie) ont permis de réunir jusqu'à 50 millions de francs, soit jusqu'à un sixième des recettes annuelles provenant de collectes.

8 Sur les composants des indicateurs et l'analyse de régression, voir aussi l'annexe. 
l'aide. C'est surtout la variable «sous-alimentation» qui détermine la répartition, tandis que la variable «catastrophe naturelle» ne semble guère avoir d'influence. Si l'on cumule, dans le cadre d'une analyse de régression linéaire, les variables «sous-alimentation» et «guerres», ces deux variables expliquent jusqu'à un quart $\left(\mathrm{r}^{2}=0,24\right)$ de la répartition géographique de l'aide privée.

Ce constat tend à renforcer l'hypothèse partielle selon laquelle l'aide privée est liée dans une certaine mesure (moins toutefois qu'on ne le pense souvent) aux catastrophes qui frappent les pays en développement. Cela étant, il reste à se demander si ce sont les catastrophes elles-mêmes ou plutôt leur médiatisation en Suisse qui déterminent le montant des dons privés 9 .

\section{$\square$ Les «pays à la mode»}

Cette hypothèse partielle suppose qu'il existe des pays en développement qui se montrent d'une part très ouverts à l'aide étrangère et qui sont d'autre part de véritables «chouchous» de l'aide internationale. Autrement dit, «les œuvres d'entraide suisses accorderaient leur aide aux pays qui en reçoivent déjà d'ailleurs », et la répartition géographique de l'aide privée suisse serait ainsi liée aux montants versés par les donateurs internationaux. A titre d'indicateur, nous utilisons ici l'aide étrangère totale dont bénéficie un pays en développement donné $^{10}$. L'analyse débouche sur un résultat surprenant. En effet, la corrélation entre les variables se révèle statistiquement significative et elle est positive. L'hypothèse est de plus renforcée par le fait que l'équation de régression explique $26 \%$ de la variance ${ }^{11}$.

\section{Facteurs propres à la Suisse}

Cette hypothèse se fonde sur l'idée que l'aide privée suisse est fortement influencée par son ancrage dans le contexte national et que cette influence devrait se retrouver dans la répartition géographique de l'aide. Ce n'est cependant ni l'éloignement des pays concernés ni l'écart linguistique et culturel qui joue un rôle dans la répartition géographique. Nous pensons en effet que c'est bien plus l'immigration, c'est-à-dire le nombre de ressortissants du pays en développement considéré vivant en Suisse, et l'aide publique de la Confédération qui déterminent le choix des pays bénéficiaires de l'aide privée.

\section{$\square$ Population immigrée provenant de pays en développement}

Voici la première hypothèse partielle: plus le nombre des ressortissants d'un pays en développement ou en transition qui vivent en Suisse est grand, plus l'aide financière allouée à ce pays sera élevée. Ce postulat peut aussi être inversé: plus l'aide financière allouée à un pays est élevée, plus le nombre de ses ressortissants à s'installer en Suisse sera grand. Nous pensons cependant que

9 Des versements importants ont par exemple été destinés à la Yougoslavie après que les médias eurent largement couvert la guerre du Kosovo, alors que le conflit de Tchétchénie, moins présent dans les médias, ne suscite que des dons modestes.

10 Sur les composants des indicateurs et l'analyse de régression, voir aussi l'annexe.

11 La corrélation apparaît aussi clairement lorsque l'on compare les vingt principaux pays bénéficiaires de l'aide mondiale et les principaux pays bénéficiaires de l'aide privée suisse (tableau 1). En effet, huit pays - Bolivie, Ethiopie, Honduras, Inde, Mozambique, Nicaragua, Tanzanie et Yougoslavie figurent sur les deux listes. 
l'influence suit la première sous-hypothèse, et ce pour les raisons suivantes. Premièrement, les personnes immigrées éveillent l'intérêt de la population suisse pour leur pays d'origine. Immigrants et Suisses apprennent à se connaître. Ce rapprochement émotionnel fait naître au sein de la population suisse la volonté de contribuer à améliorer les conditions de vie dans les pays concernés. Deuxièmement, les œuvres d'entraide peuvent s'appuyer sur les connaissances et sur l'expérience des immigrés et exploiter des synergies avec des projets menés dans leurs pays d'origine. Troisièmement, les immigrés fondent des associations pour aider la région d'où ils viennent et financer des projets de soutien en faveur des habitants de leur patrie.

A titre d'indicateur pour connaître la population résidante provenant de pays en développement ou en transition, nous utilisons les chiffres du recensement de la population réalisé en 2000 par l'Office fédéral de la statistique ${ }^{12}$.

Dans la régression linéaire, la variable «population résidante immigrée provenant des différents pays » est une variable indépendante, tandis que l'aide privée suisse allouée aux pays considérés est la variable dépendante. Le résultat de cette régression est statistiquement significatif et la corrélation est forte: la population immigrée provenant de pays du Sud et de l'Est explique $28 \%$ de la répartition géographique de l'aide privée ${ }^{13}$.

\section{$\square$ Relations entre ouvres d'entraide et Confédération (DDC)}

Nombre d'indices permettent de supposer que la répartition géographique de l'aide privée dans les pays en développement et en transition suit la répartition de l'aide publique. La principale hypothèse de cette étude est donc la suivante: les répartitions par pays de l'aide publique et de l'aide privée sont très similaires et s'influencent mutuellement. Cela signifie qu'une grande partie des fonds privés et publics destinés à la coopération au développement sont versés aux mêmes pays, autrement dit que Confédération et ouvres d'entraide travaillent avec les mêmes pays prioritaires. La coopération publique au développement exerce cependant une influence plus grande sur la coopération privée au développement que l'inverse. Voici les raisons qui nous poussent à l'affirmer.

Dans les années 1950, les initiatives privées et les activités des missionnaires ont précédé la coopération bilatérale de la Confédération. Cette situation s'explique principalement par le fait que la Suisse ne possédait pas de colonie. En 1961, la Confédération a ensuite créé un Service de la coopération technique qui était chargé de réaliser des projets bilatéraux de l'aide publique. Lors de sa création, les autorités ont prévu expressément que ses activités seraient coordonnées avec celles des œuvres d'entraide privées. La Confédération a ainsi profité de l'expérience des œuvres d'entraide, qui sont devenues les exécutants de ses projets bilatéraux. Elle a dès lors choisi de mener sa coopération bilatérale au développement avec une partie des pays dans lesquels les organismes privés étaient déjà présents ${ }^{14}$.

12 Sur les composants des indicateurs et l'analyse de régression, voir aussi l'annexe.

13 Sept pays figurent à la fois sur la liste des vingt principaux pays bénéficiaires de l'aide privée et sur celle des vingt principaux pays de provenance (pays en développement ou en transition) de la population immigrée. Voir le tableau 5 dans l'annexe.

14 Helvetas a par exemple lancé ses premiers projets au Népal en 1956. En 1962, ce pays est devenu un «pays prioritaire» de la coopération publique au développement. Au Tchad, la Confédération a 
La collaboration de la Confédération avec les œuvres d'entraide figure en bonne place dans la loi: «Le Conseil fédéral peut, dans le cadre des moyens à sa disposition, soutenir des activités d'institutions privées qui répondent aux buts formulés dans la présente loi. Ces institutions doivent y contribuer par des prestations adéquates. ${ }^{15}$

Le message concernant la continuation de la coopération de 2003 témoigne des contacts étroits qu'entretiennent la Confédération et les œuvres d'entraide: «Dès ses débuts, la coopération suisse au développement s'est distinguée par une collaboration étroite avec les organisations non gouvernementales suisses, sur les plans tant politique qu'opérationnel. $»^{16}$ De même, le message concernant la continuation de l'aide humanitaire évoque un partenariat étroit: «Les œuvres d'entraide suisses actives dans l'action humanitaire jouent un rôle important dans la mise en œuvre de projets sur le terrain. Elles partagent leurs expériences avec la DDC, et des synergies sont ainsi possibles. ${ }^{17}$

Les ONG suisses réalisent d'une part des projets et des programmes de la Direction du développement et de la coopération (DDC) (sur la base de mandats ou de contrats de régie). D'autre part, la DDC verse des contributions à des projets de développement que les œuvres d'entraide mènent de leur propre chef (treize grandes ONG bénéficient de contributions sur plusieurs années). En fin de compte, $25 \%$ à $30 \%$ de l'aide bilatérale de la DDC est mise en œuvre par les ONG suisses. Quant aux versements publics aux ONG, ils représentent $35 \%$ à $40 \%$ des activités des organisations privées ${ }^{18}$.

Depuis les années 1990, les mandats de la DDC font en général l'objet d'un appel d'offres public auquel les ONG d'autres pays et l'économie privée peuvent répondre. Cette manière de faire vise à accroître la concurrence entre les œuvres d'entraide ${ }^{19}$.

Cette collaboration étroite permet de supposer que Confédération et œuvres d'entraide choisissent de venir en aide aux mêmes pays. Lorsque la coopération publique au développement a vu le jour, les institutions privées influaient sur le choix des bénéficiaires de l'aide fédérale. Avec le temps, la coopération bilatérale de la Confédération a gagné en importance, puis le volume de cette aide a largement dépassé celui de l'aide privée. La Confédération a ainsi opéré ses choix de manière plus indépendante. Pour les œuvres d'entraide qui collaborent avec la Confédération, il est plus logique - pour exploiter les synergies potentielles - de réaliser les projets financés par des fonds privés dans les pays où

appuyé dès 1964 les activités des missionnaires suisses et ce pays est également devenu un pays prioritaire de la coopération suisse au développement.

15 Loi fédérale de 1976 sur la coopération au développement et l'aide humanitaire internationales.

16 Conseil fédéral, Message concernant la continuation de la coopération technique et de l'aide financière en faveur des pays en développement du 28 mai 2003 (FF 2003 4155), p. 4198.

17 Conseil fédéral, Message concernant la continuation de l'aide humanitaire internationale de la Confédération du 14 novembre 2001 (FF 2002 2087), p. 2110.

18 Conseil fédéral, Message concernant la continuation de la coopération technique et de l'aide financière..., op. cit., p. 4199.

19 C'est le cas par exemple dans le cadre de l'«aide aux pays de l'Est» : «[...] les mandats sont attribués sur la base d'un appel d'offres. » La Confédération contribue ainsi à «aiguiser la concurrence entre les fournisseurs». Le Conseil fédéral mentionne d'ailleurs expressément les fournisseurs étrangers (Conseil fédéral, Message sur la poursuite de la coopération renforcée avec l'Europe de l'Est et les pays de la CEI du 19 août 1998, p. 33). 
elles travaillent déjà sous régie pour la Confédération. Lancer des appels d'offres publics pour les mandats fédéraux renforce d'ailleurs cette tendance: pour se voir attribuer un mandat public, une œuvre d'entraide doit disposer d'un avantage comparatif par rapport aux autres œuvres d'entraide (et même par rapport aux services fédéraux ${ }^{20}$. L'œuvre d'entraide candidate obtiendra plus aisément un mandat si elle possède déjà une certaine expérience du pays concerné. Les œuvres d'entraide ont donc tout avantage à réaliser leurs propres projets dans les pays prioritaires de la DDC et de deviner les pays qui seront à l'avenir admis parmi les «prioritaires». Nous pensons dès lors que c'est surtout la Confédération qui exerce une influence sur le choix des pays des institutions privées et non l'inverse.

Passons à présent à l'analyse empirique. Dans ce cadre, c'est le total des prestations publiques fournies en 2001 aux pays en développement et en transition qui nous servira d'indicateur. La régression linéaire révèle un rapport positif très marqué entre l'aide publique et l'aide privée $\left(\mathrm{R}=0,71, \mathrm{r}^{2}=0,51\right)^{21}$. Ce résultat signifie que $51 \%$ de la répartition de l'aide privée s'explique par la répartition géographique de l'aide publique.

Le poids de cette corrélation apparaît aussi lorsque l'on compare les vingt principaux pays bénéficiaires de l'aide publique et les principaux pays bénéficiaires de l'aide privée (tableau 1 p. 85) : huit pays figurent sur les deux listes ${ }^{22}$. L'analyse a cependant pu être faussée par le fait que la Confédération accorde une part importante de son aide bilatérale de manière globale, sans l'attribuer à un pays précis. Tandis que seuls $10 \%$ de l'aide privée n'ont pas de destination précise, c'est le cas de $35 \%$ de l'aide publique. En raison de ce «manque de précision» du côté de la Confédération, la corrélation entre aide publique et aide privée pourrait en réalité s'avérer plus grande ou plus faible.

Graphique 5: Corrélation entre aide privée et aide publique, 2001

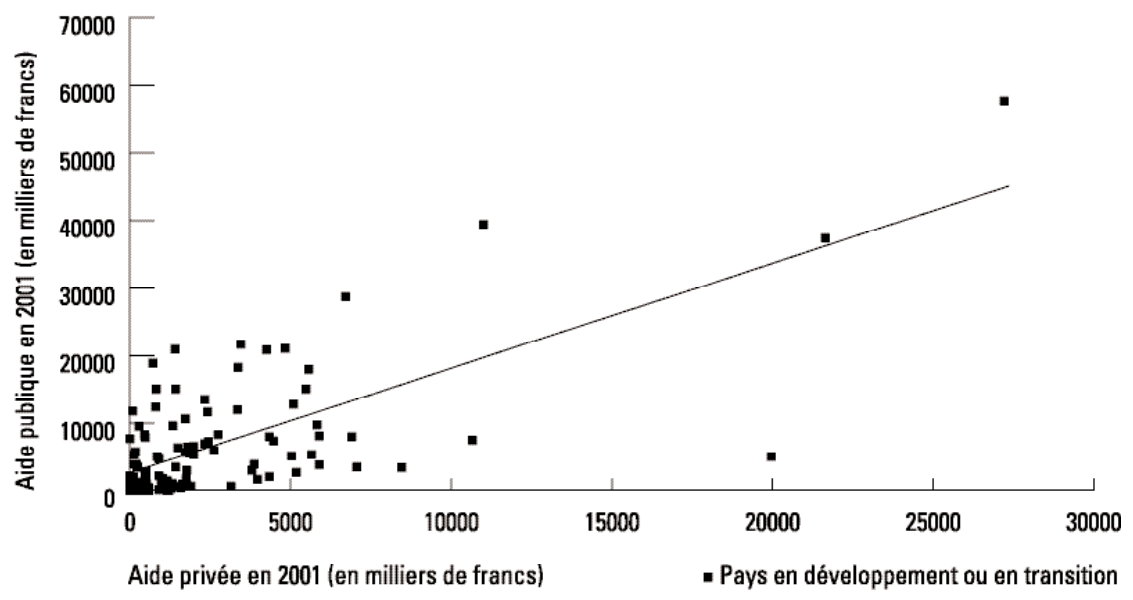

20 Il en va de même pour l'aide humanitaire: la Confédération confie des tâches aux œuvres d'entraide, notamment «lorsqu'une œuvre d'entraide dispose d'un avantage comparatif» (Conseil fédéral, Message concernant la continuation de l'aide humanitaire internationale de la Confédération, op. cit., p. 2110).

21 Voir aussi le graphique 5.

22 Voir le tableau 4 dans l'annexe. 


\section{Préférences individuelles}

C'est justement parce que l'aide privée n'est pas centralisée et qu'elle n'est pas coordonnée par une institution faîtière que les préférences individuelles jouent un rôle non négligeable dans la répartition géographique de cette aide. Ce rôle est d'autant plus important que la Suisse est un petit pays et que le montant global de l'aide privée est donc relativement modeste. Les initiatives, intérêts ou opportunités d'individus isolés (ou de petits groupes) peuvent exercer une grande influence sur la répartition géographique. Voici deux exemples marquants qui illustrent bien ce mode d'influence. En 2001, le Cambodge a reçu près de 20 millions de francs à titre d'aide privée. Ce montant correspond à $6,7 \%$ de l'aide totale et place le pays à la sixième place des pays bénéficiaires de l'aide suisse. En considérant de plus près la structure de l'aide reçue par ce pays, on constate que les seuls versements destinés à la fondation Hôpital pour enfants Kantha Bopha s'élèvent à plus de 16 millions de francs. Sans ce projet, qui repose presque entièrement sur une seule personne, ou si ce projet était mené dans un autre pays (au Laos par exemple), le Cambodge n'occuperait que la vingt-sixième place parmi les pays bénéficiaires.

Notre second exemple est l'Albanie, qui occupe le deuxième rang parmi les pays classés selon l'aide privée par habitant. Plus de $60 \%$ des versements destinés à l'Albanie proviennent de l'association Operazione Ticinese Aiuti Albania (OTAA), qui a été créée uniquement pour venir en aide à la population (catholique) du diocèse de Rreshen. Sans cette initiative d'un petit groupe de personnes, l'Albanie occuperait la neuvième place dans le classement.

Ces exemples illustrent à quel point un projet isolé ou une initiative individuelle dans un pays choisi «au hasard» peuvent influer sur la répartition géographique de l'aide. Il est néanmoins difficile de quantifier l'influence des préférences individuelles.

\section{Synthèse}

Intégrons à présent les principales variables indépendantes dans un seul modèle. C'est le facteur «aide publique» qui pèse le plus lourd. Si on lui ajoute la variable «prestations d'aide de la communauté internationale», le résultat ne change guère, de sorte que cette variable n'influe guère sur la variable dépendante «aide privée». Nous en concluons donc que la répartition de l'aide publique suit sensiblement la «mode internationale des pays bénéficiaires».

La deuxième variable par ordre d'importance est la «population immigrée», puisqu'elle représente $58 \%$ de la variable «aide publique». Comme troisième et dernier facteur indépendant, nous prenons en compte la variable «sous-alimentation» dans notre régression multiple. Son importance est encore plus faible: elle représente $53 \%$ de la variable «aide publique».

Le modèle final, incluant ces trois variables indépendantes, explique 59\% de la variable dépendante «aide privée » $\left(\mathrm{r}^{2}=0,59\right)^{23}$. Une part de $41 \%$ de la variance demeure non expliquée. Ces $41 \%$ englobent des facteurs qui nous sont inconnus ainsi que les «préférences individuelles».

23 Voir également l'annexe. 


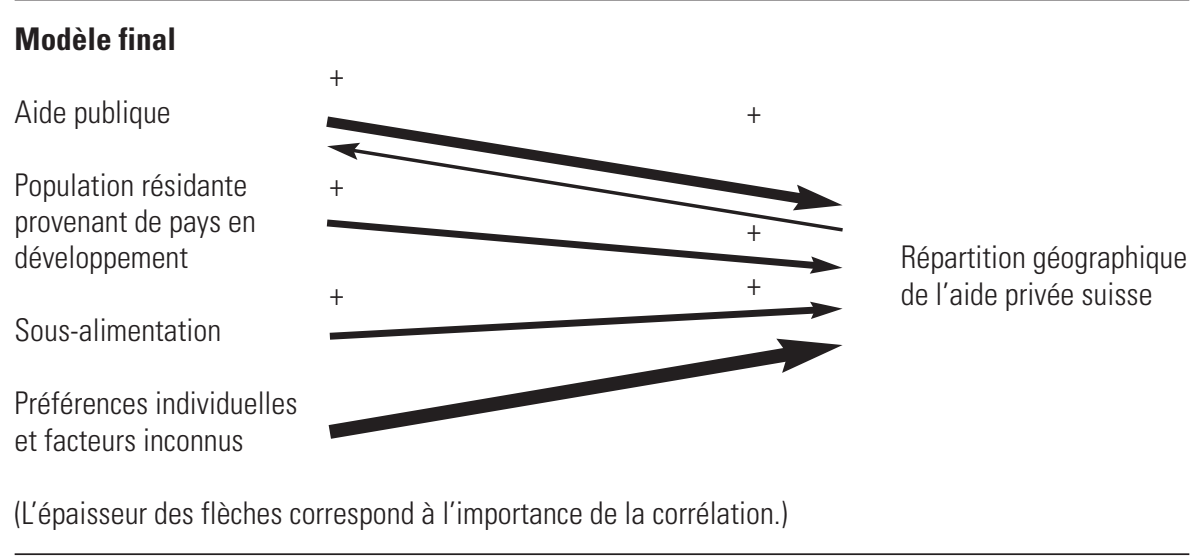

Conclusion

Nous avons vu que la répartition géographique de l'aide privée est principalement déterminée par des facteurs internes, l'aide publique étant, et de loin, le plus important d'entre eux. Pour ce qui est de leur orientation géographique, aide privée et aide publique ne sont donc pas complémentaires. Au contraire, les listes de leurs pays prioritaires se recoupent largement. Cela étant, on en vient tout naturellement à s'interroger sur la logique qui préside à la répartition de l'aide publique au développement. Quels sont les facteurs qui déterminent le choix des pays dans l'aide publique? Cette question devrait cependant faire l'objet d'une analyse séparée.

Les montants de l'aide publique et de l'aide privée subissent de grandes variations d'une année à l'autre et il serait judicieux de suivre l'évolution de ces deux variables sur plusieurs années.

Pour les raisons présentées ci-dessus, les «préférences individuelles » constituent probablement le deuxième facteur le plus important dans la répartition géographique de l'aide privée. Notre analyse quantitative ne permet toutefois pas d'explorer ce phénomène, qu'une étude qualitative permettrait de mieux cerner.

La présence en Suisse d'une population immigrée provenant de pays en développement constitue un autre facteur interne, de moindre importance toutefois, dans le choix des pays bénéficiaires.

Parmi les facteurs externes, seule la variable «sous-alimentation» pèse d'un certain poids dans la balance. Une situation humanitaire précaire dans un pays exerce en effet une influence positive sur le montant de l'aide privée suisse.

Le niveau de développement d'un pays n'exerce étonnamment aucune influence sur le montant de l'aide qui lui est attribuée. Nous avons donc dû rejeter cette hypothèse initiale. L'aide privée n'est en effet pas destinée aux pays les moins avancés. Si l'on part de l'idée que l'aide au développement devrait s'adresser en premier lieu aux pays les plus pauvres, force est de qualifier l'aide privée d'incohérente. 
Par ailleurs, les guerres et les catastrophes naturelles n'influent guère sur l'aide privée. Il conviendrait cependant de se pencher ici sur le rôle des médias et d'analyser dans quelle mesure la médiatisation d'une catastrophe ou d'une guerre détermine le montant de l'aide privée allouée aux pays touchés.

Les résultats de notre analyse permettent de répondre comme suit à la question de la logique du choix des pays bénéficiaires de l'aide privée: la répartition géographique de l'aide privée suisse dépend beaucoup de la Suisse et très peu des pays en développement ou en transition.

\section{SOURCES}

Allan Pierre, «La complexité, le hasard et l'individu dans la théorie politique internationale», in Girard Michel (dir.), Individu et politique internationale, Paris, Economica, 1994.

FAO, The State of Food Insecurity in the World 2003, Rome, 2003.

Gerster Richard, Nord-Süd-Politik: abschreiben oder investieren?, Zürich, Orell Füssli Verlag, 1995.

Grüninger Beat, «Unsere tägliche Katastrophe gib uns heute... », Mosquito, nº 4, Juni 1997.

Landolf Daniel, Fankhauser Michael, Gekaufte Entwicklungshilfe?, Köniz, Edition Soziothek, 1996.

Le Monde diplomatique, «La diffusion des langues multi-étatiques», in L'Atlas du Monde diplomatique, Paris, 2003.

PNUD, Rapport mondial sur le développement humain 2002, Bruxelles, Editions De Boeck Université, 2002.

Conseil fédéral, Message concernant la continuation de la coopération technique et de l'aide financière en faveur des pays en développement du 7 décembre 1998.

Conseil fédéral, Message concernant la continuation de la coopération technique et de l'aide financière en faveur des pays en développement du 28 mai 2003 (FF 2003 4155).

Conseil fédéral, Message concernant la continuation de l'aide humanitaire internationale de la Confédération du 14 novembre 2001 (FF 2002 2087).

Conseil fédéral, Message sur la poursuite de la coopération renforcée avec l'Europe de l'Est et les pays de la CEI du 19 août 1998.

DDC, Aide de la Suisse aux pays en développement et aux pays en transition - statistiques 2001, Berne, DDC, 2003.

DDC, Rapport annuel de la coopération internationale de la Suisse - 2001, Berne, DDC, 2002.

DDC, Service statistique, Berne.

Office fédéral de la statistique, Recensement de la population de 2000 (chiffres concernant la population résidante étrangère), Neuchâtel, 2002.

Banque mondiale, GRICS (Governance Research Indicator Country Snapshot) - Composite Governance Indicator 2000, <http://info.worldbank.org/governance/kkz2002/govmap.asp>.

Chaîne du Bonheur, rapports annuels de 1998 à 2001, Berne.

Freedom House, Freedom in the World 2000-2001, <www.freedomhouse.org/research/freeworld/2001/ index.htm>.

OCDE, Statistical Annex of the 2003 Development Cooperation Report, Paris, 2004.

SIPRI, FIRST (Facts on International Relations and Security Trends), Major Armed Conflicts 1997-2001, $<$ http://first.sipri.org $>$.

SwissRe, Société suisse de réassurance (éd.), Catastrophes naturelles et techniques, années 1998-2001, sigma, Zurich.

\section{SITES INTERNET}

Fondation Kantha Bopha: <www.beat-richner.ch>.

Operazione Ticinese Aiuti Albania: <www.otaa.ch>. 


\section{Annexe}

\section{A) Variables}

1. Aide privée en provenance de la Suisse destinée à des pays en développement ou en transition $\rightarrow$ variable dépendante "aide privée "

Pour quantifier cette notion, nous utilisons les chiffres d'un sondage réalisé en 2001 sur les fonds privés versés par des fondations, des associations, des œuvres d'entraide ou par d'autres organismes suisses à des partenaires dans des pays en développement ou en transition, pour mener des activités d'utilité publique dans les domaines de la coopération au développement ou de l'aide humanitaire ${ }^{24}$.

\section{Variable dépendante "aide privée par habitant"}

Les données obtenues au point 1 sont divisées par le nombre d'habitants du pays en développement ou en transition considéré.

3. Niveau de développement d'un pays donné $\rightarrow$ variable indépendante «niveau de développement"

Pour chiffrer cette notion, nous nous servons de l'indicateur synthétique de I'ONU de 2000, qui quantifie le niveau du "développement humain» (incluant l'espérance de vie, la formation et le pouvoir d'achat par habitant) dans les pays concernés. Cet indicateur va de 0 (développement humain très faible) à 1 (développement humain très élevé) ${ }^{25}$.

4. Situation humanitaire dans un pays $\rightarrow$ elle comprend les variables indépendantes " guerres ", " catastrophes naturelles " et " sous-alimentation "

\subsection{Quantification des "guerres"}

Pour évaluer la gravité d'un conflit armé, nous nous fondons sur le nombre des victimes tel qu'il a été enregistré pour les années 1997 à 2001 par l'Institut international de recherche sur la paix (SIPRI), sis en Suède. Les données disponibles n'étant pas toujours complètes et précises, nous avons défini un indicateur allant de 0 (absence de conflit) à 4 (conflit armé très violent).

\subsection{Quantification des "catastrophes naturelles"}

Pour évaluer la gravité des catastrophes naturelles, nous utilisons les indications sur le nombre des victimes de catastrophes naturelles publiées pour les années 1998 à 2001 par la Société suisse de réassurance (SwissRe) $)^{26}$. Nous n'avons accordé qu'un facteur réduit de moitié au nombre de victimes des années 1998 et 1999, car nous admettons qu'une partie de la reconstruction avait débuté avant 2001. De même, nous n'avons prévu qu'un facteur réduit de moitié pour les catastrophes de la seconde moitié de 2001, car nous supposons que la reconstruction (et le flux financier qui lui est inhérent) n'a débuté qu'avec un certain retard.

\subsection{Quantification de la "sous-alimentation"}

Nous nous servons ici des données réunies par la FAO sur le nombre des personnes souffrant de sousalimentation pendant la période 1999-200127.

5. Le montant de l'aide qu'un pays reçoit de l'étranger $\rightarrow$ variable indépendante "pays à la mode"

Pour quantifier cette variable, nous utilisons les données récoltées par I'OCDE en 2001 sur le montant total de l'aide qu'un pays en développement ou en transition donné a obtenu de l'étranger28. 
6. Personnes originaires de pays en développement ou en transition résidant en Suisse $\rightarrow$ variable indépendante "population immigrée"

La quantification de cette variable utilise les informations sur l'origine de la population résidante suisse réunies par l'Office fédéral de la statistique lors du recensement de la population de $2000^{29}$.

7. Aide publique dans les pays en développement ou en transition $\rightarrow$ variable indépendante "aide publique"

Cette variable est quantifiée sur la base des flux financiers bilatéraux provenant du secteur public suisse en 2001, tels qu'ils ont été recensés par la DDC. (Ces flux comprennent l'aide publique au développement et l'aide publique) $)^{30}$.

\section{B) Régressions ${ }^{31}$}

1. Niveau de développement d'un pays

Variable dépendante: "aide privée par habitant»

Variable indépendante: «niveau de développement»

$\mathrm{R}=0,094 \quad \mathrm{r}^{2}=0,009 \quad$ Statistiquement insignifiant

2. Situations de détresse et aspects humanitaires

Variable dépendante: "aide privée»

Variable indépendante: "guerres", "sous-alimentation"

$R=0,488 \quad r^{2}=0,238 \quad$ Statistiquement significatif au seuil de $1 \%$

"Guerres " : bêta $=0,249 \quad$ Statistiquement significatif au seuil de $1 \%$

"Sous-alimentation" : bêta $=0,365 \quad$ Statistiquement significatif au seuil de $1 \%$

3. Les «pays à la mode » dans l'aide privée

Variable dépendante: "aide privée»

Variable indépendante: "pays à la mode»

$R=0,512 \quad r^{2}=0,263 \quad$ Statistiquement significatif au seuil de $1 \%$

4. Population immigrée provenant de pays en développement ou en transition

Variable dépendante: "aide privée»

Variable indépendante: "population étrangère "

$R=0,526 \quad r^{2}=0,277 \quad$ Statistiquement significatif au seuil de $1 \%$

5. Relations entre œuvres d'entraide et Confédération (DDC)

Variable dépendante: "aide privée»

Variable indépendante: "aide publique»

$R=0,711 \quad r^{2}=0,506 \quad$ Statistiquement significatif au seuil de $1 \%$

\section{Synthèse}

Variable dépendante: "aide privée»

Variables indépendantes: "aide publique», "population immigrée ", "sous-alimentation "

$R=0,768 \quad r^{2}=0,590 \quad$ Statistiquement significatif au seuil de $1 \%$

"Aide publique» : bêta $=0,475 \quad$ Statistiquement significatif au seuil de $1 \%$

"Population immigrée» : bêta $=0,277 \quad$ Statistiquement significatif au seuil de $1 \%$

"Sous-alimentation» : bêta $=0,252 \quad$ Statistiquement significatif au seuil de $1 \%$

29 Office fédéral de la statistique, Recensement de la population de 2000 (chiffres concernant la population résidante étrangère), Neuchâtel, 2002.

30 DDC, Aide de la Suisse aux pays en développement et aux pays en transition..., op. cit.

$31 \mathrm{R}=$ coefficient de corrélation, $\mathrm{r}^{2}=$ coefficient de détermination linéaire, bêta = coefficient de régression standardisé (indique l'importance des variables indépendantes concernées dans le modèle global). 


\section{C) Tableaux}

Les pays inscrits en gras figurent également parmi les vingt pays vers lesquels convergent les montants les plus élevés de l'aide privée (tableau 1, "aide en chiffres absolus", pour les tableaux 4, 5 et 6; tableau 2, "aide par habitant", pour le tableau 3).

Tableau 3: "Niveau de développement " - Les vingt pays les moins avancés, 2000 (indice de développement humain)

\begin{tabular}{llll}
\hline 1. Sierra Leone & 0.275 & 11. Rép. centrafricaine & 0.375 \\
\hline 2. Niger & 0.277 & 12. Mali & 0.386 \\
\hline 3. Afghanistan* & 0.300 & 13. Liberia* & 0.400 \\
\hline 4. Burundi & 0.313 & 14. Malawi & 0.400 \\
\hline 5. Mozambique & 0.322 & 15. Angola & 0.403 \\
\hline 6. Burkina Faso & 0.325 & 16. Rwanda & 0.403 \\
\hline 7. Ethiopie & 0.327 & 17. Gambie & 0.405 \\
\hline 8. Guinée-Bissau & 0.349 & 18. Guinée & 0.414 \\
\hline 9. Somalie* & 0.350 & 19. Bénin & 0.420 \\
\hline 10. Tchad & 0.365 & 20. Erythrée & 0.421 \\
\hline
\end{tabular}

* Nos propres estimations.

Tableau 4: "Aide publique » - Les vingt principaux bénéficiaires de l'aide publique au développement, 2001 (en milliers de francs)

\begin{tabular}{llll}
\hline 1. Yougoslavie & 57741 & 11. Bolivie & 17979 \\
\hline 2. Mozambique & 39393 & 12. Russie & 15112 \\
\hline 3. Inde & 37399 & 13. Pérou & 15095 \\
\hline 4. Tanzanie & 28725 & 14. Pakistan & 15046 \\
\hline 5. Burkina Faso & 21687 & 15. Afrique du Sud & 13552 \\
\hline 6. Bosnie-Herzégovine & 21078 & 16. Afghanistan & 12771 \\
\hline 7. Népal & 20912 & 17. Niger & 12378 \\
\hline 8. Bangladesh & 20790 & 18. Tchad & 12027 \\
\hline 9. Bulgarie & 18869 & 19. Tadjikistan & 11765 \\
\hline 10. Vietnam & 18262 & 20. Equateur & 11688 \\
\hline
\end{tabular}

Tableau 5: «Population immigrée» - Les vingt principaux pays d'origine des immigrés, 2001 (nombre de personnes)

\begin{tabular}{|c|c|c|c|}
\hline 1. Yougoslavie & 213524 & 11. Thaillande & 5722 \\
\hline 2. Turquie & 83312 & 12. Maroc & 5680 \\
\hline 3. Macédoine & 55714 & 13. Philippines & 4986 \\
\hline 4. Bosnie-Herzégovine & 47286 & 14. Vietnam & 4791 \\
\hline 5. Croatie & 43084 & 15. Somalie & 4764 \\
\hline 6. Sri Lanka & 32708 & 16. Pologne & 4689 \\
\hline Brésil & 8611 & 17. Irak & 4567 \\
\hline Russie & 6812 & 18. Rép. dominicaine & 4486 \\
\hline 9. Chine & 6315 & 19. Tunisie & 4418 \\
\hline 10. Inde & 6142 & 20. Rép dém. du Congo & 4168 \\
\hline
\end{tabular}

Tableau 6: "Sous-alimentation » - Les vingt pays les plus touchés par la sous-alimentation, 1999-2001 (en millions de personnes sous-alimentées)

\begin{tabular}{|c|c|c|c|}
\hline 1. Inde & 213.7 & 11. Vietnam & 15.1 \\
\hline 2. Chine & 135.3 & 12. Indonesie & 12.6 \\
\hline 3. Bangladesh & 44.1 & 13. Thaillande & 11.9 \\
\hline 4. Rép dém. du Congo & 38.3 & 14. Kenya & 11.5 \\
\hline 5. Pakistan & 26.8 & 15. Mozambique & 9.7 \\
\hline 6. Ethiopie & 26.4 & 16. Nigeria & 9.1 \\
\hline 7. Philippines & 16.8 & 17. Soudan & 7.7 \\
\hline 8. Brésil & 15.6 & 18. Rép. dém. de Corée & 7.5 \\
\hline 9. Afghanistan & 15.3 & 19. Angola & 6.4 \\
\hline 10. Tanzanie & 15.2 & 20. Ouzbékistan & 6.4 \\
\hline
\end{tabular}

